\title{
Analysis of High Frequency Fetal Heart Rate Variability Using Empirical Mode Decomposition
}

\author{
MR Ortiz $^{1}$, ER Bojorges ${ }^{1}$, SD Aguilar $^{1}$, JC Echeverría $^{1}$, R González-Camarena $^{2}, \mathrm{~S} \mathrm{Carrasco}^{2}$, \\ MJ Gaitán ${ }^{2}$, A Martínez ${ }^{1}$ \\ ${ }^{1}$ Electrical Engineering Department \\ ${ }^{2}$ Health Science Department \\ Universidad Autónoma Metropolitana-Izt, Mexico City, Mexico
}

\begin{abstract}
We used Empirical Mode Decomposition (EMD) to assess the high frequency information of fetal HRV during different conditions of fetal activity. Beat-to-beat data of thirteen normal fetuses at term (36 to 40 gestation weeks) were obtained from abdominal ECG signals. In addition, corporal and breathing fetal movements were simultaneously observed using ultrasound B mode images. High frequency components of fetal HRV data were obtained by applying the EMD and by reconstructing the signals from components having frequencies higher than $0.3 \mathrm{~Hz}$, according to the lowest frequency of fetal breathing movements observed in our data. Our results show significant differences in the power of high frequency components for episodes only with breathing movements vs no movements and vs. episodes having corporal movements $(p<0.05)$. In conclusion, EMD seems to be a useful tool to analyze the effect of the FBM on non-stationary fetal HR time series.
\end{abstract}

\section{Introduction}

Fetal heart rate (FHR) patterns are generally used to assess fetal well-being. These patterns could show changes depending on pathological conditions as hypoxia and fetal distress, but also on physiological states of normal fetus as age and fetal behavior [1]. During fetal corporal movements (FCM), for example, FHR patterns change generally causing accelerations (fetal reactivity) and it has been associated to healthy fetuses [2]. It also has been observed that fetal breathing movements (FBM) cause that short term variability of fetal heart rate increases, and it has been associated to respiratory sinus arrhythmia (RSA) [3, 4, 5]. Fetal RSA analysis has been suggested as a useful tool to evaluate nervous development [6, 7]. As FBM and FCM affect FHR and fetal heart rate variability (FHRV) and their incidence is variable (depending on multiple factors as age, blood glucose level, etc.), it is necessary to consider them on FHRV analysis.

Different methods to analyze FHRV have been proposed, most of them based on long- and short-term FHRV indexes. These indexes are usually obtained from time and frequency domain data after removing accelerations and decelerations and they are frequently based on statistical and spectral analysis respectively $[8,9]$. However, the reliability of the techniques used for this analysis depends on the stationarity of the signals.

Empirical Mode Decomposition (EMD) is a useful method to analyze non-stationary and non-linear time series and it has been used on HRV analysis for long-term adult records [10]. Given the non-stationary nature of FHR time series, the aim of the present study was to explore the detection of fetal RSA by using EMD.

\section{Methodology}

Twenty-four abdominal ECG records were obtained from thirteen pregnant women ( $24 \pm 3$ years old) with fetal gestation ages between 36 and 40 weeks and low to middle risk factors. In addition, fetal corporal and breathing movements were simultaneously observed by using ultrasound B mode images and indications of each type of movement were generated. All the registers were made between 8:30 and 10:30 hours, with time period after patient breakfast less than three hours. Subjects provided their informed consent to participate in the study.

\subsection{General procedure}

Beat-to-beat fetal HRV data were obtained from five minutes abdominal ECG signals using a build on tested algorithm. In order to generate indications of FBM, one ultrasound video observer keep holding a marker during the time where fetal "inspiratory efforts" were present, other- 


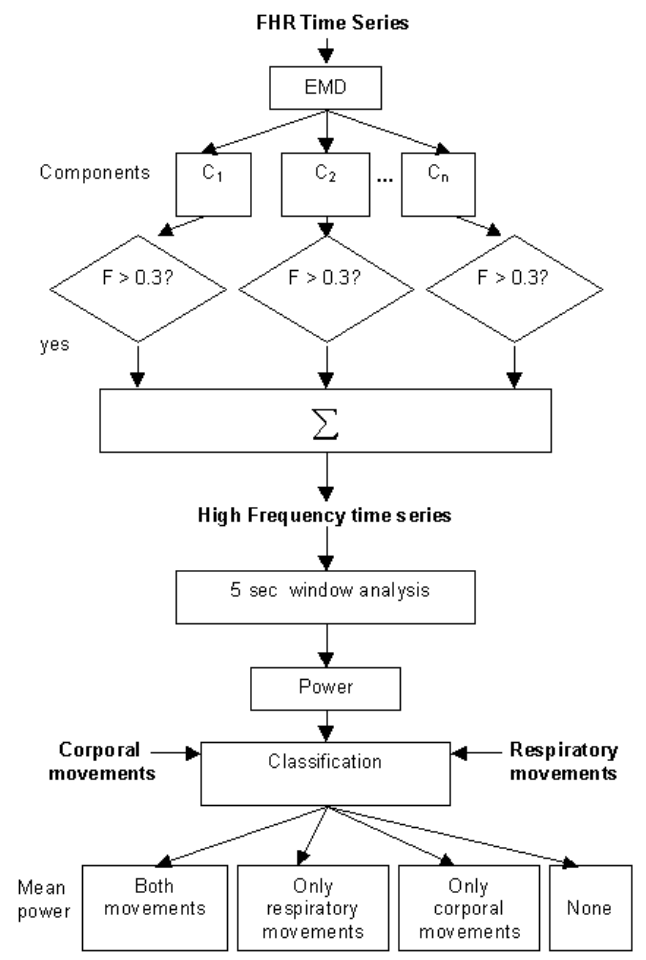

Figure 1. Scheme of the method proposed for data analysis

wise it was released. In the same way, other observer generated indications corresponding to each FCM by pressing another marker when corporal movements were identified. Thus, the high value of binary indications corresponded to corporal movements or "inspiratory" effort presence and low value to absence of corporal or breathing movements.

High frequency information of FHR time series were obtained by applying the EMD (see below) and by reconstructing the signal from high frequency components. The power of reconstructed signals was analyzed using five seconds length window. Windows were classified according to the fetal activity in one of four types of episodes: 1) with both kind of movements, 2) just with breathing movements, 3) only with corporal movements or 4) with none of them. Finally, the mean power of each condition, were compared against the condition with FBM , because it was considered as control. This process is shown in figure 1 .

\subsection{Empirical mode decomposition}

The decomposition method [11] is essentially based on the identification of the extremes of the original time series to apply a sifting procedure in which upper and lower envelopes are created by a cubic spline interpolation of the local maxima, and local minima, respectively. The difference between the original data $X(t)$ and the mean $m_{1}$ ob- tained from the upper and lower envelopes is designated as the first potential component $C_{1 p}$

$$
C_{1 p}=X(t)-m_{1}
$$

Ideally, this component will be an Instantaneous Mode Function (IMF), which becomes suitable for defining a meaningful instantaneous frequency [11]. Yet new extremes can be generated, with the existing ones being either shifted or exaggerated. Thus, the sifting procedure must be applied more than once to eliminate the riding waves and to achieve a more symmetrical wave-profile by smoothing the uneven amplitudes. According to Huang et al. [11], a threshold should be computed by summing the squared normalized amplitude differences from two consecutive sifting results, between 0.2 and 0.4 as a possible limit to obtain the desired IMF first component $\left(C_{1}\right)$. Once this condition is reached, the first component $C_{1}$ is subtracted from the original series $X(t)$ to leave the first residue, $R_{1}$

$$
R_{1}=X(t)-C_{1}
$$

As this residue still contains information for longer scales, the decomposition procedure described above is then successively applied to obtain the next component. Each time a new component is reached, the new residual information is employed as the input information for the continuation of the procedure. Hence, the decomposition performs a general separation of the original signal into locally non-overlapping time scale components.

\subsection{High frequency analysis}

EMD was applied to original beat-to-beat FHR time series to obtain its components. After a spectral analysis, of each component, those with frequency content higher than $0.3 \mathrm{~Hz}$ were selected to reconstruct the high frequency signal. This limit value was selected according to the lowest FBM frequency identified in our data.

Once high frequency signals were reconstructed, the power was computed every 5 seconds, using overlapped windows. These windows were delayed by one sample, in order to follow consecutive changes on power value due to episodic behavior of fetal activity. The equation 3 shows how power was computed, while figure 2 presents the resultant signals after applying this sequence to one record.

$$
p(x)=\frac{\sum_{i}^{n} x_{i}^{2}}{n}
$$

where $x$ is the signal to analyze (in this case high frequency FHRV), $i$ means the sample index and $n$ is the window length. 

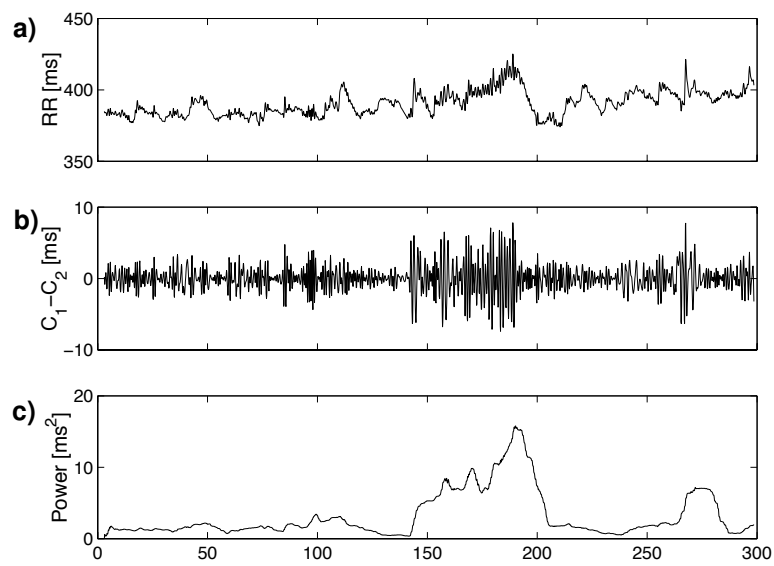

Figure 2. a) FHR time series of one fetus. b) Reconstructed signal using the high frequency components. c) Windowed power using continuous $5 \mathrm{sec}$ windows

\subsection{Classification}

According to fetal activity, windows were classified as without FBM when episodes of breathing akinesia ("apnea") were present for periods longer than $50 \%$ of the window length, otherwise, they were classified as having FBM. Given that fetal heart rate responds to a FCM with an acceleration (fetal reactivity) and that it can persist even when the FCM have finished, we used a fifteen seconds time delay after a corporal movement finished to establish the end of this condition. So, all the windows that match with the time from the beginning of the FCM marker to the end of the time delay described above, were considered as with FCM. This delay time was suggested by empirical experience according to fetal reactivity observed. The combination of these two types of movements gave us the four possible fetal activity conditions described in General procedure.

\subsection{Data analysis}

Three of the thirteen subjects were not consider on the analysis because they did not present one or more conditions of fetal activity described above. This gave us twenty records to be analyzed.

After window classification, the mean power for each condition was computed per patient as a parameter to assess the effect of fetal activity on FHRV. ANOVA test for repeated measures was used to compare the mean power among the conditions. Bonferroni's with control post hoc analysis was performed to established significant differences. Condition with only FBM was used as control on statistical test. A $p<0.05$ was assumed as statistical significance.

\section{Results}

In all the cases $C_{1}$ was used to reconstruct the high frequency signals, while $C_{2}$ and $C_{3}$ were added in some cases.

Descriptive analysis showed that condition having both movements, rejected the normality assumption, so it was discarded from the Bonferroni's test. Mean power of high frequency FHRV, for the other three conditions were: $13.22 \pm 7.90 \mathrm{~ms}^{2}$ for episodes having only FBM; 7.72 \pm 5.25 for periods only with FCM, and $7.59 \pm 5.24$ for the quiet group (see figure 3 ). These results show higher values for episodes with FBM vs. those without movements and vs. those with FCM $(P<0.05)$. Figure 4 presents an example of the power behavior related to the four mentioned conditions. As it can be seen, the mean power of the high frequency FHR increases when the fetus presents FBM (condition 2 on the figure, and it decreases on quiet sate (condition 1 on the figure). Power graphic on figure 4 also shows that there is an increase on the mean power associated neither to FBM nor to FCM but to acceleration/deceleration presence (see figure 2 and figure 4).

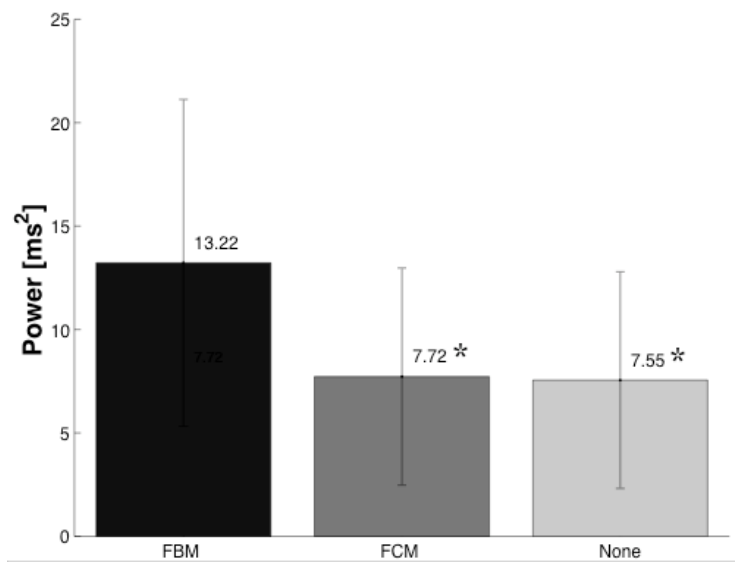

Figure 3. All patients mean power bars for the conditions compared. *, means significant differences in comparison with FBM condition.

\section{Discussion}

The behavior of the high frequency FHR power was analyzed using the EMD method. The analysis was made on 10 fetuses at term for four different fetal activity conditions and by using five seconds window analysis in order to follow de consecutive changes on the power due to fetal activity. Our main findings were: when fetus presents only FBM the mean power raise respect the basal state (without movements); and respect FCM condition. This allows 
a)

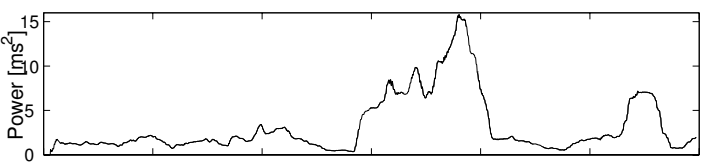

b)

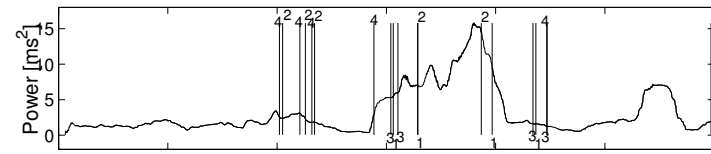

c)

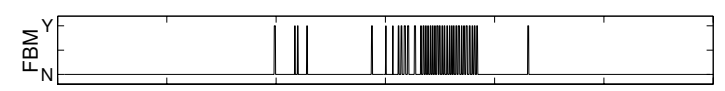

d)

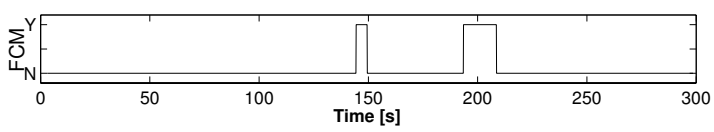

Figure 4. a) Power using $5 \mathrm{sec}$ continuous window. b) Classification of power signal, lines indicate the end of each condition and numbers from 1 to 4 meaning: both movements, only Fetal Respiratory Movements (FRM), only Fetal Body Movements (FBM) and none of them, respectively. c) FRM marker. d) FBM marker.

establishing that an increment on the mean power of high frequency FHR is associated to FBM activity and it could be a useful method to analyze RSA as it was established before $[3,4,12,7,5]$.

We also observed that mean power of high frequency FHR time series could increase during abrupt and large accelerations or decelerations periods, even when there were not FBM or FCM, so it could be useful to eliminate this type of segments before the analysis. We expected that presence of FCM would reduce short term variability because of the fetal reactivity, but our results show no differences among FCM and quiet fetal state. These results can be explained by considering that the delay time after the ending of FCM for classifying the fetal activity condition could change depending on the type and magnitude of the corporal movement. So it could be recommendable to consider only large corporal movements instead of all of them during the generation of the FCM indications by the observer, since they do produce accelerations.

\section{Conclusions}

For spotting differences between FBM and quiet conditions, EMD seems to be a useful tool to analyze the effect of the FBM on non-stationary FHR time series. The EMD method allows an extraction of intrinsic high frequency information from non stationary FHR time series, in contrast with the traditional spectral analysis which reliability depends on the stationarity of the signal.

\section{Acknowledgements}

The support of volunteer mothers, Dr. Carlos Vargas and CIMIGen is gratefully acknowledged.

\section{References}

[1] van Woerden E.E., van Geijn H. A critical appraisal of fetal surveillance. Amsterdam, London, New York, Tokyo: Excerpta Medica, 1994; 211-220.

[2] Manning F. Fetal biophysical profile. Obstet Gynecol Clinics of North America 1999;26:557-577.

[3] Timor-Tritsch I, Zador I, Hertz R, Rosen M. Human fetal respiratory arrhythmia. Am J Obstet Gynecol 1977; 127:662-666.

[4] Divon M, Zimmer E, Platt L, Paldi E. Human fetal breathing: Associated changes in heart rate and beat-to-beat variability. Am J Obstet Gynecol 1985;151:403-406.

[5] Donchin Y, Caton D, Porges S. Spectral analysis on fetal heart rate in sheep: The occurrence of respiratory sinus arrhythmia. Am J Obstet Gynecol 1984;148:1130-1135.

[6] Dipietro J, Hodgson D, K.A. C, S.C. H. Fetal neurobehavioral development. Child Development 1996;67:25532567.

[7] Wakai R. Assessment of fetal neurodevelopment via fetal magnetocardiography. Experimental Neurology 2004; 190:S65-S71.

[8] Parer W, Parer J, Holbrook R, Block S. Validity of mathematical methods of quantitating fetal heart rate variability. Am J Obstet Gynecol 1985;153:402-409.

[9] Cerruti S, Civardi S, Signorini M, Ferrazi E, Pardi G, Bianchi A. Spectral analysis of antepartum heart rate variability. Clin Phys Physiol Meas 1989;10:27-31.

[10] Echeverría J, J.A. C, M.S. W, Hayes-Gill BR. Application of empirical mode decomposition to heart rate variability analysis. Med Biol Eng Comput 2001;39:471-479.

[11] Huang NE, Shen Z, Long S, Wu M, Shih H, Zheng Q, Tung C, H.H. L. The empirical mode decomposition and the hilbert spectrum for non linear and non stationary time series analysis. Royal Society London 1998;903-995.

[12] Dawes G, Visser G, Goodman J, Levine D. Numerical analysis of the human fetal heart rate: modulation by breathing and movement. Am J Obstet Gynecol 1981;140:535-544.

Address for correspondence:

MR Ortiz

UAM-I, Ingeniería Eléctrica

San Rafael Atlixco 186, 09340, México DF, México

mrop@xanum.uam.mx 